\title{
Aortic arch types and postoperative outcomes after carotid artery stenting in asymptomatic and symptomatic patients
}

\author{
Renato CASANA ${ }^{1,2}$ *, Daniele BISSACCO ${ }^{3}$, Chiara MALLOGGI 2 , Valerio S. TOLVA ${ }^{4}$, Andrea ODERO JR ${ }^{1}$, \\ Maurizio DOMANIN 3, , Santi TRIMARCHI 3, 5, Vincenzo SILANI 6, 7, Gianfranco PARATI 8, 9
}

${ }^{1}$ Department of Surgery, Istituto Auxologico Italiano IRCCS, Milan, Italy; ${ }^{2}$ Laboratory of Research in Vascular Surgery, Istituto Auxologico Italiano IRCCS, Milan, Italy; ${ }^{3}$ Unit of Vascular Surgery, Maggiore Polyclinic IRCCS, Milan, Italy; ${ }^{4}$ Department of Vascular and Endovascular Surgery, Polyclinic of Monza, Monza, Italy; ${ }^{5}$ University of Milan, Milan, Italy; ${ }^{6}$ Department of Neurology-Stroke and Neuroscience, Istituto Auxologico Italiano IRCCS, San Luca Hospital, Milan, Italy; ${ }^{7}$ Department of Pathophysiology and Transplantation, University of Milan, Milan, Italy; ${ }^{8}$ Department of Cardiovascular, Neural and Metabolic Sciences, Istituto Auxologico Italiano, IRCCS, San Luca Hospital, Milan, Italy; ${ }^{9}$ Department of Medicine and Surgery, University of Milano-Bicocca, Monza, Italy

*Corresponding author: Renato Casana, Department of Surgery, Istituto Auxologico Italiano IRCCS, Via Mercalli 30, 20122 Milan, Italy. E-mail: r.casana@auxologico.it

\section{A B STRACT}

Background: The aim of this study was to investigate the influence of the aortic arch type on technical and clinical success of carotid artery stenting (CAS) procedure.

Methods: Clinical and anatomical data of consecutive patients who underwent CAS from 2010 to 2018 were prospectively collected and retrospectively analyzed. Primary outcome was technical success, define as successful stent delivery and deployment and $<30 \%$ residual carotid stenosis. Secondary outcomes were death, stroke, myocardial infarction (MI) and transient ischemic attack (TIA) rates at 30 days after CAS. Subgroups analysis with asymptomatic and symptomatic patients were also performed.

Results: During the study period, 523 patients were enrolled and analyzed. Among these, $176(33.6 \%)$ had Type I, 227 (43.4\%) had Type II and $120(23.0 \%)$ had Type III or bovine aortic arch (BAA) type. Technical success rate was achieved in $96.0 \%$ of cases. At 30 days, if compared with Type I or II, patient with Type III or BAA experienced a higher death rate ( 0 vs. 0 vs. $1.8 \%$, respectively; $\mathrm{P}=0.056)$ and combined postoperative stroke/TIA rate (3\% vs. $2.8 \%$ vs. $9.9 \%$, respectively; $\mathrm{P}=0.012)$. No differences for same outcomes between asymptomatic and symptomatic patients were described, although the latter group experienced more postoperative MI. A multivariate analysis revealed Type III or BAA as an independent risk factor for postoperative stroke/TIA (HR 3.23 , IC95\% 1.40-7.45; $\mathrm{P}=0.006$ ).

Conclusions: In this cohort of patients, death and postoperative neurological complications rates were associated with Type III or BAA, irrespective of symptomatic patients' status. Extremely attention is required during perioperative period in patients who were candidate to CAS and with challenging aortic arch anatomy. 
(Cite this article as: Casana R, Bissacco D, Malloggi C, Tolva VS, Odero Jr A, Domanin M, et al. Aortic arch types and postoperative outcomes after carotid artery stenting in asymptomatic and symptomatic patients. Int Angiol 2020;39:000-000. DOI: 10.23736/S0392-9590.20.04494-6)

Key words: Carotid artery diseases; Carotid arteries; Stents; Stroke; Aorta, thoracic.

$\mathrm{T}$ he role of carotid artery stenting (CAS) in preventing ischemic stroke is showing increasingly convincing perioperative and long-term results, 1 with a lower incidence of cranial nerve palsy and myocardial infarction (MI), thanks to the improvement in endovascular techniques and the increase in operator experience. 2,3

Evidence suggests that systemic factors and medical comorbidities $^{4-6}$ may have a prognostic impact on the adverse event rate for carotid endarterectomy whereas local anatomic and lesions factors increase the technical difficulties $^{7}$ and risk of neurologic complications ${ }^{8}$ associated with CAS.

There is growing evidence that the ability of the operator impacts on the effectiveness of carotid stenting in achieving minimal complications and preventing stroke. ${ }^{9}$

The severity of carotid stenosis, the degree of tortuosity, ${ }^{10}$ and the aortic arch type $8,11,12$ have been identified as possible causes of technical failure.

In fact, aortic arch type plays an important role in determining the technical success rate and perioperative risk of complications. Difficult aortic arch may require repeated aggressive manipulation, which may generate emboli. Moreover, unfavorable arch anatomy increases the difficulty in guiding catheter or long sheath insertion.

Complex aortic arch anatomy is recurrent in the population. It has been estimated that up to $10 \%$ of the population presented with bovine aortic arch, in which the left common carotid artery originates from the brachiocephalic trunk, rather than arising directly from the aortic arch as a separate branch as occurs in the most common aortic arch branching patterns. ${ }^{13}$

However, data on the impact of aortic arch anatomy on technical success and clinical outcome are sparse and patient selection and exclusion criteria differed among the studies.

The purpose of this study was to investigate the influence of the aortic arch anatomy on the technical and clinical success of carotid artery stent implantation.

\section{Materials and methods}

\section{Patient population}

The clinical data of all consecutive patients who underwent CAS for carotid revascularization from 2010 to 2018 were analyzed retrospectively. Data were recorded prospectively. Indications for CAS were a carotid artery diameter reduction of at least $50 \%$, in case of symptomatic patients, or $\geq 80 \%$, in case of asymptomatic ones, diagnosed by head and neck computer tomography angiography (CTA) or magnetic resonance angiography (MRA) using the North American Symptomatic Carotid Endarterectomy Trial (NASCET) criteria. ${ }^{14}$ To describe aortic arch type, maximum intensity projection (MIP) and multiplanar reconstruction (MPR) technique were used. To characterize aortic arch type, for each patient the diameter of the left common carotid artery (LCCA) and the arch height were measured, according to current literature recommendations. ${ }^{15,} 16$ An arch height $<1$ time the LCCA diameter indicates a Type I arch, 1-2 times a Type II and $>2$ times a Type III arch. Bovine aortic arch (BAA) was defined as a common origin for the innominate and left common carotid arteries or other variants. ${ }^{17}$

All patients provided written informed consent. The study was approved by the local Ethics committee.

\section{CAS procedure}

As preoperative evaluations, physical examination baseline assessment, carotid duplex ultrasound scan (DUS) of the supra-aortic vessels and a neurological assessment measured using the National Institutes of Health Stroke Scale (NIHSS) were performed. Dual antiplatelet therapy was administered within 24 hours before the procedure. All patients received a loading dose of clopidogrel (300 $\mathrm{mg}$ ) one hour before the procedure. CAS treatments were performed following an established internal protocol which has been previously described. ${ }^{18}$ All procedures were done in the operating room, equipped with a portable imaging fluoroscopic C-arm (OEC 9900 elite; GE Medical Siemens, Wisconsin), by two skilled operators with a high 
volume experience ( $>50 \mathrm{CEA} / \mathrm{CAS}$ procedures per year as first operator). Iodinated or gadolinium contrast was used in patients with normal creatinine level or creatinine $>1.5$ $\mathrm{mg} / \mathrm{dL}(132 \mathrm{mmol} / \mathrm{L})$, respectively. Intraoperative anticoagulation was achieved using an intravenous unfractionated heparin bolus (100-units/kg heparin) in order to ensure an activated clotting time (ACT) $>250 \mathrm{~s}$ throughout the procedure. All patients were treated with percutaneous transfemoral approach, under DUS control. The right common femoral artery was preferred, although femoral calcifications may change this preference.

Open-cell, closed-cell, hybrid and micromesh carotid stents were employed, as a function of lesion characteristics and vessel anatomy. Temporary distal or proximal cerebral protection was performed in all patients during the procedure using a Emboshield NAV6 system (Abbott Vascular, Green Oaks, IL, USA) or a Mo.Ma system (Medtronic, Minneapolis, MN, USA), respectively. The lesion was post dilated using a 4.5- or 5.5-mm diameter Rx Viatrak 14 Plus balloon (Abbott Vascular, Green Oaks, IL, USA).

Medical therapy continued with $100 \mathrm{mg}$ of aspirin once daily for a lifelong period and $75 \mathrm{mg}$ clopidogrel once daily for 1 month after CAS procedure.

\section{Definitions and endpoints}

Primary endpoint was intraoperative CAS success rate, in terms of technical achievement. Technical success was defined as efficacious delivery and deployment of the carotid stent at the carotid target lesion site, with $<30 \%$ residual stenosis. Secondary endpoints were death, stroke, TIA and MI rate within 30 days after intervention, in the entire population, and depending on arch type. Subgroups analysis were also performed to test differences in asymptomatic and symptomatic patients.

Minor strokes were defined as neurological deficits $(\mathrm{NIHSS} \leq 4)$, which resolved completely within 30 days or those which did not cause chronic functional impairment in daily activities, as assessed by the neurologist. Otherwise, stroke was defined as major neurological damage (NIHSS $>4)$. All patients affected by postoperative neurological complications was submitted to postoperative brain computed tomography and neurological evaluation to better assess injury entity and gravity.

\section{Statistical analysis}

Categorical variables were presented as number and percentages and were compared using the Fisher exact test. Continuous variables were presented as mean \pm standard deviations (SD) and were compared using the Student's $t$ test. Multivariate analysis was carried out to examine the independent associations among procedural outcomes and patients baseline characteristics. All probability values were two-sided and a value of $\mathrm{P} \leq 0.05$ was considered statistically significant. All analyses were performed using STATA $^{\mathrm{TM}}$ (STATA Corp., version 14.0, College Station, TX, USA).

\section{Results}

In total, 523 patients were included in the analysis, 329 (62.9\%) asymptomatic and 194 (37.1\%) symptomatic. Baseline characteristics of the study participants are summarized in Table I, II, divided according to arch type and symptomatic status, respectively. A majority of the patients presented with Type II aortic arch $(\mathrm{N} .=227,43.4 \%)$. Type I and Type III or BAA aortic arch were present in $33.7 \%(\mathrm{~N} .=176)$ and $16.4 \%(\mathrm{~N} .=86)$ of the patients, respectively. Finally, BAA was present in $6.5 \%(\mathrm{~N} .=34)$ of the patients. Patients with Type III or BAA were most likely to be smokers $(43.3 \%$ vs. $31.3 \%$ and $29.0 \%$ in Type II and I, respectively; $\mathrm{P}=0.03$ ), and to have chronic obstructive pulmonary disease (COPD, $35.0 \%$ vs. $10.6 \%$ vs. $14.8 \% ; \mathrm{P}<0.01)$. On the contrary, patients with Type I arch were most likely to be symptomatic $(55.1 \% v s .24 .2 \%$ and $35.0 \%$ in Type II and III+BBA, respectively; $\mathrm{P}<0.01$ ), and to have had a congestive heart failure (CHF, $17.0 \%$ vs. $7.5 \%$ and $15.8 \%$ in Type II and III or BAA, respectively; $\mathrm{P}<0.01$ ). Patients with Type II arch were most likely to

\begin{tabular}{lrccr}
\multicolumn{3}{c}{ TABLE I._Baseline characteristics according to arch type. } \\
\cline { 2 - 5 } Characteristic & \multicolumn{4}{c}{ Aortic arch type } \\
\cline { 2 - 5 } & \multicolumn{1}{c}{ Type I } & Type II & Type III+BAA & \multicolumn{1}{c}{ P } \\
\hline Age $\geq 80$ & $93(52.5)$ & $111(48.9)$ & $66(55.0)$ & 0.520 \\
Gender (M) & $100(56.8)$ & $140(61.7)$ & $74(61.7)$ & 0.570 \\
Smokers & $51(29.0)$ & $71(31.3)$ & $52(43.3)$ & 0.030 \\
Symptomatic & $97(55.1)$ & $55(24.2)$ & $42(35.0)$ & $<0.001$ \\
Diabetes & $64(36.4)$ & $74(32.6)$ & $51(42.5)$ & 0.190 \\
Hyperlipidemia & $138(78.4)$ & $167(73.6)$ & $94(78.5)$ & 0.460 \\
Coronary artery & $53(30.1)$ & $62(27.3)$ & $31(25.8)$ & 0.710 \\
$\quad$ disease & & & & \\
Hypertension & $136(77.3)$ & $192(84.6)$ & $83(69.2)$ & $<0.001$ \\
Chronic obstructive & $26(14.8)$ & $24(10.6)$ & $42(35.0)$ & $<0.001$ \\
$\quad$ pulmonary disease & & & & \\
History of stroke & $23(13.1)$ & $70(30.8)$ & $37(30.8)$ & $<0.001$ \\
Chronic heart failure & $30(17.0)$ & $17(7.5)$ & $19(15.8)$ & $<0.001$ \\
History of myocardial & $36(20.5)$ & $34(15.0)$ & $30(25.0)$ & 0.072 \\
$\quad \begin{array}{l}\text { infarction } \\
\text { Total }\end{array}$ & $176(33.6)$ & $227(43.4)$ & $120(23.0)$ & \\
\hline
\end{tabular}

Values are expressed as N. (\%)

BAA: bovine aortic arch. 
TABLE II.-Baseline characteristics according to symptomatic status.

\begin{tabular}{lrrr} 
Characteristic & Asymptomatic & Symptomatic & \multicolumn{1}{c}{$P$} \\
\hline Gender (M) & $204(62.0)$ & $110(56.7)$ & 0.270 \\
Smokers & $91(27.7)$ & $83(42.8)$ & $<0.001$ \\
Aortic arch type & & & \\
$\quad$ Type I & $79(24.0)$ & $97(50.0)$ & $<0.001$ \\
$\quad$ Type II & $171(52.0)$ & $55(28.4)$ & $<0.001$ \\
$\quad$ Type III+BAA & $79(24.0)$ & $42(21.6)$ & 0.590 \\
Age $\geq 80$ & $164(49.8)$ & $106(54.6)$ & 0.170 \\
Diabetes & $102(31.0)$ & $87(44.8)$ & 0.002 \\
Hyperlipidemia & $266(80.9)$ & $133(68.6)$ & 0.001 \\
Coronary artery disease & $75(22.8)$ & $71(36.6)$ & 0.001 \\
Hypertension & $245(74.5)$ & $166(85.6)$ & 0.003 \\
Chronic obstructive pulmonary & $47(14.3)$ & $45(23.2)$ & 0.012 \\
$\quad$ disease & & & \\
History of stroke & $79(24.0)$ & $51(26.3)$ & 0.600 \\
Chronic heart failure & $43(13.1)$ & $23(11.9)$ & 0.780 \\
History of myocardial infarction & $75(22.8)$ & $25(12.9)$ & 0.006 \\
Total & $329(62.9)$ & $194(37.1)$ & \\
\hline
\end{tabular}

Values are expressed as N. (\%)

BAA: bovine aortic arch.

TABLE III.-Intraoperative characteristics according to arch type.

\begin{tabular}{lcccc} 
& \multicolumn{5}{c}{ Aortic arch type } \\
\cline { 2 - 4 } Characteristic & Type I & Type II & Type III+BAA & P \\
\hline $\begin{array}{l}\text { Target vessel } \\
\text { (right) }\end{array}$ & $92(52.3)$ & $133(58.6)$ & $104(86.7)$ & $<0.001$ \\
Stent type & & & & \\
$\quad$ Open cell stent & $59(33.5)$ & $103(45.4)$ & $92(76.7)$ & $<0.001$ \\
Closed cell stent & $99(56.3)$ & $42(18.5)$ & $0(0.0)$ & $<0.001$ \\
Hybrid stent & $17(9.7)$ & $52(22.9)$ & $1(0.8)$ & $<0.001$ \\
$\quad$ Micromesh stent & $1(0.6)$ & $30(13.2)$ & $27(22.5)$ & $<0.001$ \\
X-ray time & $737.5 \pm 345.7$ & $763.5 \pm 491.4$ & $814.1 \pm 365.8$ & 0.641 \\
$\quad$ (s, mean $\pm S D)$ & & & & \\
X-ray dose & $93.7 \pm 71.8$ & $75.4 \pm 40.5$ & $80.1 \pm 57.0$ & 0.329 \\
$\quad$ (mGy, mean $\pm S D)$ & & & & \\
Technical failure & $5(2.8 \%)$ & $6(2.6 \%)$ & $10(8.3 \%)$ & 0.042 \\
Total & $176(33.6)$ & $227(43.4)$ & $120(23.0)$ & \\
\hline
\end{tabular}

Values are expressed as N. (\%)

BAA: bovine aortic arch.

have hypertension if compared with Type I and III or BAA ( $84.6 \%$ vs. $77.3 \%$ and $69.2 \%$, respectively; $\mathrm{P}<0.01)$. No statistically significant differences were found in the other demographic and clinical characteristics among the aortic arch types. Regarding differences between asymptomatic and symptomatic group, the latter had more Type I and less Type II arch. Furthermore, symptomatic patients experienced more comorbidities.

Angiographic and procedural characteristics are summarized in Table III. The mean fluoroscopy time did not differ among the three groups, ranging from a mean value of $737.5 \pm 345.7 \mathrm{~s}$ in Type I aortic arch to a mean value of
$814.1 \pm 365.8 \mathrm{~s}$ in Type III or BAA. No significant differences were found in the $\mathrm{x}$-ray dose according to the aortic arch type. The greatest dose was used in Type I aortic arches $(93.7 \pm 71.8 \mathrm{mGy})$, and the lowest dose was used in Type II aortic arches (75.4 \pm 40.5 mGy).

Technical success rate could be achieved in $96.0 \%$ of cases (502 patients). The procedures were suspended and/ or converted to open surgery mostly due to the difficult anatomy of the aortic arch configuration, which made it impossible the deployment of the stent. Technical failures due to arch tortuosity was higher in Type III or BAA, compared with Type I and II ( $8.3 \%$ vs. $2.6 \%$ vs. $2.8 \%$, respectively; $\mathrm{P}=0.042$ ).

The all 30-day adverse events are shown in Table IV. Sixteen adverse events occurred in patients with Type III or BAA arch, including two deaths $(1.8 \%)$, seven strokes $(6.3 \%)$, three MIs $(2.7 \%)$, and four TIAs $(3.6 \%)$. The incidence of postoperative neurological accidents was higher in patients with Type III or bovine arches with respect to those with Type I or II arches (10.0\% vs. $2.9 \%$ vs. $2.7 \%$, respectively; $\mathrm{P}=0.012$ ).

Dividing patients in asymptomatic and symptomatic status, the group with preoperative neurological symptoms experienced more postoperative MI but no postoperative neurological complications, if compared with asymptomatic ones (Table V, VI).

Multivariate analysis revealed Type III or BAA as an independent risk factor for postoperative stroke/TIA (Hazard Ratio, HR 3.23, 95\% Confidence Interval, IC95\% 1.40-

TABLE IV.-Thirty-day outcomes according to arch type.

\begin{tabular}{lcccc} 
& \multicolumn{4}{c}{ Aortic arch type } \\
\cline { 2 - 5 } Outcome & Type I & Type II & Type III+BAA & P \\
\hline Death & $0(0.0)$ & $0(0.0)$ & $2(1.8)$ & 0.056 \\
Myocardial infarction & $4(2.3)$ & $4(1.8)$ & $3(2.7)$ & 0.795 \\
Postoperative neurological & $5(3.0)$ & $6(2.8)$ & $11(9.9)$ & 0.012 \\
$\quad$ complications & & & & \\
Total & $176(33.6)$ & $227(43.4)$ & $120(23.0)$ & \\
\hline
\end{tabular}

Values are expressed as N. (\%)

BAA: bovine aortic arch.

TABLE V.-Thirty-day outcomes according to symptomatic status.

\begin{tabular}{lccc} 
Outcome & Asymptomatic & Symptomatic & $P$ \\
\hline Death & $0(0.0)$ & $2(1.1)$ & 0.149 \\
Myocardial infarction & $2(0.6)$ & $9(4.8)$ & 0.003 \\
Postoperative neurological & $10(3.2)$ & $12(6.5)$ & 0.113 \\
$\quad$ complications & $329(62.9)$ & $194(37.1)$ & \\
Total &
\end{tabular}

Values are expressed as N. (\%). 


\begin{tabular}{|c|c|c|c|}
\hline & Adjusted HR & $95 \% \mathrm{Cl}$ & $\mathrm{P}$ \\
\hline Гype I aortic arch & 0.37 & $0.12-1.14$ & 0.084 \\
\hline Type II aortic arch & 0.34 & $0.12-0.98$ & 0.046 \\
\hline Туре III+BAA & 3.23 & $1.40-7.45$ & 0.006 \\
\hline Symptomatic & 1.73 & $0.70-4.27$ & 0.232 \\
\hline Hypertension & 1.19 & $0.39-3.62$ & 0.758 \\
\hline COPD & 1.83 & $0.72-4.68$ & 0.203 \\
\hline History of stroke & 2.65 & $1.05-6.71$ & 0.039 \\
\hline Target vessel, right & 2.14 & $0.78-5.88$ & 0.14 \\
\hline Stent type & 1.11 & $0.75-1.66$ & 0.59 \\
\hline
\end{tabular}

7.45; $\mathrm{P}=0.006)$. Furthermore, also a history of stroke was independently associated with postoperative neurological complications (HR 2.65, IC95\% 1.05-6.71; $\mathrm{P}=0.039$ ).

\section{Discussion}

Aortic arch type remains a well-known preoperative characteristic that may predict postoperative outcomes after CAS. This report was design to evaluate if difficult arch anatomy (Type III or BAA) remains and independent predictor of postoperative neurological complications, death and MI, irrespective of symptomatic or asymptomatic patients' preoperative status.

As first important findings, although Type III or BAA type was found as an independent risk factor for poor postoperative outcomes, no differences were evaluated in term of X-ray time and dose during CAS. Indirectly, although it was not analyzed, operation time may be supposed similar among arch type groups. This data differs from previously published data. Shen et al. ${ }^{15}$ found that Type III aortic arches were associated with a longer fluoroscopy time and higher contrast agent dose, in a group of 224 patients presenting with Type I, II and III aortic arch types. Such results were confirmed by other findings suggesting that angiographic characteristics and the tortuosity of the target vessel were associated with increased fluoroscopic time, which reflects a technically challenging procedure. 19,20 Discrepancy between this and others comparative studies may be explained by the great variability in X-ray dose and time within each group (standard deviations in the majority of case exceeded the half of average value) and surgeon experience which minimized radiation exposure also in case of challenging cases. ${ }^{21}$ On the other hand, although not statistically significant difference is visible and probably is not consistent due to the relative low sample size of groups.

Despite these, technical failure remains much more present in case of arch Type III or BAA.
Macdonald and coworkers developed a scoring system to identify expected difficulties during CAS procedure, based on a consensus among several specialists, such as interventional radiologists, neuroradiologists, interventional cardiologists and vascular surgeons. ${ }^{22} \mathrm{~A}$ different study proposed a standardized method for quantifying tortuosity in carotid arteries, by adopting angulation measurements used in other anatomic areas. ${ }^{10}$ In this study, only proximal tortuosity was found to be significantly associated with successful deployment of stent in the target lesion and risk of neurologic intraoperative and postoperative complications.

Although it did not increase intraoperative time, challenging anatomy and technical difficulty remain related with poor postoperative outcomes, not only in terms of neurological complications, but also for death. In literature, a significant relationship has been found between the incidence of stroke and the catheterization difficulties, especially when less experienced operators performed the procedures. ${ }^{23}$ It is known that CAS is a technically demanding procedure, with a steep "learning curve"24-27. It has been previously reported suboptimal CAS results caused by low operator experience. It is worth noting that all the procedures of the present study were conducted by the same surgeon team with a high CAS volume and extensive experience.

During CAS, the aortic arch and the common carotid artery must be manipulated and traversed to reach the diseased carotid artery. Thus, the increased incidence of perioperative complications during CAS in Type III or BAA group may be related to the unfavorable vascular anatomy. ${ }^{11,15,16}$ Furthermore, despite a higher percentage of right treated carotid artery was described in Type III or BAA patients (suggesting a "longer way" to target carotid artery as a risk factor), no significant correlation with postoperative outcomes was finally obtained after multivariate analysis.

This personal retrospective analysis on the relationship between aortic arch type and perioperative complications have change drastically our approach in patients with unfavorable anatomy. At this time, all patients with Type III or BAA are treated with transcervical approach. Four patients ( 3 male and 1 female, all asymptomatic) were treated with this approach, with no perioperative complications (unpublished data).

Transcervical approach, with or without flow reversal, has been established as a safe and valid method in patients with unfavorable anatomy, to avoid aortic arch and the risk of increase embolization rate. ${ }^{28,29}$ Furthermore, a recent in- 
vestigation from the Society for Vascular Surgery Vascular Quality Initiative (SVS-VQI) demonstrated the superiority of transcervical compared with transfemoral CAS. ${ }^{30}$ Due to non-univocal and robust results, in our daily practice, a transcervical approach remains reserved only in patients with relative or absolute contraindication to transfemoral access. This also because a low perioperative complication rate, a validated operative protocol and CAS operators skilled experience with transfemoral approach have been obtained among years.

Several potential limitations concerning our study findings need to be addressed. First, it is a retrospective study and the potential patient selection and treatment bias may not be excluded. Second, diffusion weighted magnetic resonance imaging (DW-MRI) and transcranial doppler were not performed in this cohort, so the incidence of new ischemic brain lesions from the target lesion or embolic signal in the basal arteries of the brain were not evaluated. Third, only short-term (30 days) adverse events were collected, although the prognostic effects of intraprocedural technique may be associated also with mid-term or longterm complications.

\section{Conclusions}

In this cohort of unselected consecutive patients, although there were no preoperative and intraprocedural differences between patients with arch Type I, II and III or BAA, the latter group experienced more postoperative cerebrovascular events. A careful patient selection, based on arch anatomy and history of cerebrovascular accidents, and well-experienced operators are pivotal to reduce postoperative complications rate during CAS procedures.

\section{References}

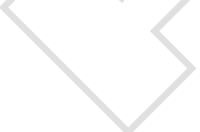

1. Spanos K, Karathanos C, Lachanas VA, Drakou A, Stamoulis K, Koutsias S, et al. Real-world experience of extracranial carotid artery interventions for atherosclerotic disease during a 10-year period. Int Angiol 2018;37:465-70

2. Brott TG, Hobson RW 2nd, Howard G, Roubin GS, Clark WM, Brooks $\mathrm{W}$, et al.; CREST Investigators. Stenting versus endarterectomy for treatment of carotid-artery stenosis. N Engl J Med 2010;363:11-23.

3. Meier P, Knapp G, Tamhane U, Chaturvedi S, Gurm HS. Short term and intermediate term comparison of endarterectomy versus stenting for carotid artery stenosis: systematic review and meta-analysis of randomised controlled clinical trials. BMJ 2010;340:c467.

4. Casana R, Malloggi C, Odero A Jr, Tolva V, Bulbulia R, Halliday A, et al. Is diabetes a marker of higher risk after carotid revascularization? Experience from a single centre. Diab Vasc Dis Res 2018;15:314-21.

5. Casana R, Malloggi C, Tolva VS, Odero A Jr, Bulbulia R, Halliday $\mathrm{A}$, et al. Does metabolic syndrome influence short and long term durability of carotid endarterectomy and stenting? Diabetes Metab Res Rev 2019;35:e3084.
6. Casana R, Tolva VS, Odero A Jr, Malloggi C, Silani V, Parati G. Carotid artery stenting is safe and effective for symptomatic patients with acute coronary syndrome. Catheter Cardiovasc Interv 2020;96:129-35.

7. Choi HM, Hobson RW, Goldstein J, Chakhtoura E, Lal BK, Haser PB, et al. Technical challenges in a program of carotid artery stenting. J Vasc Surg 2004;40:746-51, discussion 751.

8. Lin SC, Trocciola SM, Rhee J, Dayal R, Chaer R, Morrissey NJ, et al. Analysis of anatomic factors and age in patients undergoing carotid angioplasty and stenting. Ann Vasc Surg 2005;19:798-804.

9. Connors JJ 3rd, Sacks D, Furlan AJ, Selman WR, Russell EJ, Stieg $\mathrm{PE}$, et al.; NeuroVascular Coalition Writing Group; American Academy of Neurology; American Association of Neurological Surgeons; American Society of Interventional and Therapeutic Radiology; American Society of Neuroradiology; Congress of Neurological Surgeons; AANS/CNS Cerebrovascular Section; Society of Interventional Radiology. Training, competency, and credentialing standards for diagnostic cervicocerebra angiography, carotid stenting, and cerebrovascular intervention: a joint statement from the American Academy of Neurology, American Association of Neurological Surgeons, American Society of Interventional and Therapeutic Radiology, American Society of Neuroradiology, Congress of Neurological Surgeons, AANS/CNS Cerebrovascular Section, and Society of Interventional Radiology.. Radiology 2005;234:26-34.

10. Faggioli G, Ferri M, Gargiulo M, Freyrie A, Fratesi F, Manzoli L, et al. Measurement and impact of proximal and distal tortuosity in carotid stenting procedures. J Vasc Surg 2007;46:1119-24.

11. Faggioli GL, Ferri M, Freyrie A, Gargiulo M, Fratesi F, Rossi C, et al. Aortic arch anomalies are associated with increased risk of neurological events in carotid stent procedures. Eur J Vasc Endovasc Surg 2007;33:436-41.

12. Hobson RW 2nd, Howard VJ, Roubin GS, Brott TG, Ferguson RD, Popma JJ, et al.; CREST Investigators. Carotid artery stenting is associated with increased complications in octogenarians: 30-day stroke and death rates in the CREST lead-in phase. J Vasc Surg 2004;40:1106-11.

13. Shaw JA, Gravereaux EC, Eisenhauer AC. Carotid stenting in the bovine arch. Catheter Cardiovasc Interv 2003;60:566-9.

14. Barnett HJ, Taylor DW, Haynes RB, Sackett DL, Peerless SJ, Ferguson GG, et al.; North American Symptomatic Carotid Endarterectomy Trial Collaborators. Beneficial effect of carotid endarterectomy in symptomatic patients with high-grade carotid stenosis. N Engl J Med 1991;325:445-53.

15. Shen S, Jiang X, Dong H, Peng M, Wang Z, Che W, et al. Effect of aortic arch type on technical indicators in patients undergoing carotid artery stenting. J Int Med Res 2019;47:682-8.

16. Burzotta F, Nerla R, Pirozzolo G, Aurigemma C, Niccoli G, Leone $\mathrm{AM}$, et al. Clinical and procedural impact of aortic arch anatomic variants in carotid stenting procedures. Catheter Cardiovasc Interv 2015;86:480-9. 17. Layton KF, Kallmes DF, Cloft HJ, Lindell EP, Cox VS. Bovine aortic arch variant in humans: clarification of a common misnomer. AJNR Am J Neuroradiol 2006;27:1541-2.

18. Casana R, Tolva V, Odero A Jr, Malloggi C, Paolucci A, Triulzi F, et al. Safety and efficacy of the new micromesh-covered stent CGuard in patients undergoing carotid artery stenting: early experience from a single centre. Eur J Vasc Endovasc Surg 2017;54:681-7.

19. Madhwal S, Rajagopal V, Bhatt DL, Bajzer CT, Whitlow P, Kapadia SR. Predictors of difficult carotid stenting as determined by aortic arch angiography. J Invasive Cardiol 2008;20:200-4.

20. Bendszus M, Koltzenburg M, Burger R, Warmuth-Metz M, Hofmann E, Solymosi L. Silent embolism in diagnostic cerebral angiography and neurointerventional procedures: a prospective study. Lancet 1999;354:1594-7.

21. Myrcha P, Miłek T, Woźniak W, Kozdój B, Ciostek P. 3D-fusionimaging-assisted carotid artery stenting is safe and feasible. Int Angiol 2019;38:326-33.

22. Macdonald S, Lee R, Williams R, Stansby G; Delphi Carotid Stenting Consensus Panel. Towards safer carotid artery stenting: a scoring system for anatomic suitability. Stroke 2009;40:1698-703.

23. Gray WA, Yadav JS, Verta P, Scicli A, Fairman R, Wholey M, et al.; 
CAPTURE Trial Collaborators. The CAPTURE registry: predictors of outcomes in carotid artery stenting with embolic protection for high surgical risk patients in the early post-approval setting. Catheter Cardiovasc Interv 2007; 70:1025-33.

24. Nallamothu BK, Gurm HS, Ting HH, Goodney PP, Rogers MA, Curtis JP, et al. Operator experience and carotid stenting outcomes in Medicare beneficiaries. JAMA 2011;306:1338-43.

25. Huibers A, Calvet D, Kennedy F, Czuriga-Kovács KR, Featherstone RL, Moll FL, et al. Mechanism of Procedural Stroke Following Carotid Endarterectomy or Carotid Artery Stenting Within the International $\mathrm{Ca}-$ rotid Stenting Study (ICSS) Randomised Trial. Eur J Vasc Endovasc Surg 2015;50:281-8.

26. Bijuklic K, Tübler T, Wandler A, Goossens-Merkt H, Schofer J. Carotid artery stenting, what can be learned after more than 1,000 patients: a single centre single operator experience. EuroIntervention 2011;7:820-7.
27. Bonati LH, Dobson J, Algra A, Branchereau A, Chatellier G, Fraedrich $\mathrm{G}$, et al.; Carotid Stenting Trialists' Collaboration. Short-term outcome after stenting versus endarterectomy for symptomatic carotid stenosis: a preplanned meta-analysis of individual patient data. Lancet 2010;376:1062-73.

28. Christopoulos D, Philippov E, Kallintzi M. Safe carotid artery angioplasty and stenting in patients unsuitable for transfemoral approach. Int Angiol 2010;29:37-40.

29. Paraskevas KI, de Borst GJ, Eckstein HH, Schermerhorn ML. Transfemoral vs Transcervical Carotid Artery Stenting. J Endovasc Ther 2019;26:228-30.

30. Malas MB, Dakour-Aridi H, Wang GJ, Kashyap VS, Motaganahalli RL, Eldrup-Jorgensen J, et al. Transcarotid artery revascularization versus transfemoral carotid artery stenting in the Society for Vascular Surgery Vascular Quality Initiative. J Vasc Surg 2019;69:92-103.e2.

Conflicts of interest.-The authors certify that there is no conflict of interest with any financial organization regarding the material discussed in the manuscript.

Funding.-This research was funded by Istituto Auxologico Italiano IRCCS, CARIVASC project, Italian Ministry of Health, "Ricerca Corrente 2019" under Grant 34C901_2019.

Authors' contributions. - Renato Casana gave substantial contributions to study design, to the performance of procedures and to manuscript revision; Daniele Bissacco contributed to manuscript revision; Chiara Malloggi contributed to data collection, data analysis and manuscript draft; Valerio S. Tolva and Andrea Odero Jr contributed to the performance of procedures; Maurizio Domanin, Santi Trimarchi, Vincenzo Silani and Gianfranco Parati contributed to manuscript revision. All authors read and approved the final version of the manuscript.

History.-Article first published online: October 21, 2020. - Manuscript accepted: October 14, 2020. - Manuscript revised: September 28, 2020. - Manuscript received: June 29, 2020. 